\title{
Comunidades de Aprendizaje en Siglo XXI
}

\author{
Erika Coto-Jiménez \\ Universidad de Iberoamérica \\ Xinia L. Calvo-Mora \\ Melissa González-Castro \\ Universidad Técnica Nacional \\ Christian Peñaranda-Castro \\ Ministerio de Educación Pública \\ Konrad Sauter-Echeverría \\ Universidad Autónoma de Centro América
}

\section{Resumen:}

Este artículo indaga sobre las comunidades de aprendizaje como forma eficaz de aprender en grupo. En específico, la investigación se centra en situaciones de aprendizaje en la educación superior en Latinoamérica. La inquietud primordial que tenían los autores era repasar experiencias del sistema de aprendizaje en el programa de doctorado en educación de la Universidad De La Salle en San José, Costa Rica fundado por el Dr. Francisco Gutiérrez hace más de 20 años y del cuál se han graduado más de 600 personas provenientes en su mayoría de Iberoamérica. Se ha escrito mucho sobre comunidades de aprendizaje a nivel mundial durante varias décadas, sin embargo, los autores quisieron cuestionarse la metodología de aprendizaje en la que han estado inmersos por tres años; buscar las experiencias de otros aprendientes del programa doctoral en el pasado; y comparar situaciones similares reportadas en revistas académicas iberoamericanas.

Palabras clave: Comunidades de aprendizaje, Paradigma Emergente, Entramado, Educación, Aprendizaje, Creatividad

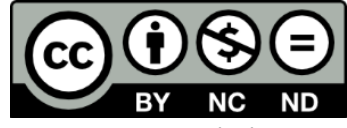




\section{Introducción}

Es frecuente identificar evidencia de que nuestros ancestros, los sapiens, vivían usualmente en grupos. Harari (2018), menciona que las especies que evolucionaron de ancestros comunes, como lo somos los Homo sapiens, pertenecen a una familia en común, lo cual nos sugiere la convivencia e interacción en agrupaciones afines. Esto nos permite comprender que el concepto de comunidad de aprendizaje cobra sentido desde los primeros grupos humanos nativos y que la cooperación estaba presente para lograr "metas- objetivos comunes". Desde ese entonces, se narraban las experiencias que las comunidades iban acumulando a través de las prácticas sociales en donde el conocimiento era un acto de participación. Sin embargo, las comunidades actuales de aprendizaje tienen sus cimientos a principios de la humanidad. El gran maestro Paulo Freire, influyó en Latinoamérica mestiza como promotor para que esta tendencia tuviera el auge que hoy posee. Pretendemos en este apartado comenzar a delimitar el concepto de comunidad de aprendizaje (C.A.) desde diferentes perspectivas.

Cuando reflexionamos sobre ¿qué implica?, o ¿cómo se concibe una comunidad de aprendizaje? consideramos oportuna la propuesta que comparten Castillo y Ramírez (2016) donde las autoras definen como "COMUNIDAD APRENDIENTE" a aquella que se nutre del encuentro genuino, sin jerarquías, [en donde] todas las personas que pertenecen a la comunidad son portadores de sentidos, aprendizajes y pasiones. La horizontalidad en las relaciones favorece la posibilidad de compartir y construir aprendizajes" (p. 4)

Las comunidades de aprendizaje no buscan únicamente centrarse en el qué y cómo se aprende, sino que incluyen también el quién, el dónde y para qué de los procesos de aprender y conocer. Reconoce la heterogeneidad y coexistencia de diferentes teorías y prácticas que pretenden lograr una revisión profunda sobre la educación y los procesos que esta conlleva (Coll, 2001). Como autores y autoras, después de un largo camino en el doctorado universitario en Educación de la Universidad La Salle de Costa Rica, nos preocupamos por plasmar en este artículo teórico el sentir, el compartir conocimiento y el cómo estas emociones permiten aprendizajes libres, creativos y más continuos en las comunidades de aprendizaje. Deseamos compartir con los y las lectoras de este artículo teórico las diferentes experiencias y estrategias para promover, generar espacios, ambientes en donde el acto de aprender se vive en el seno de la misma comunidad aprendiente y se resignifican nuevas formas de entender la misma comunidad y el contexto vital de cada persona, lo cual implica el cambio de mirada que deseamos los una en comunidad.

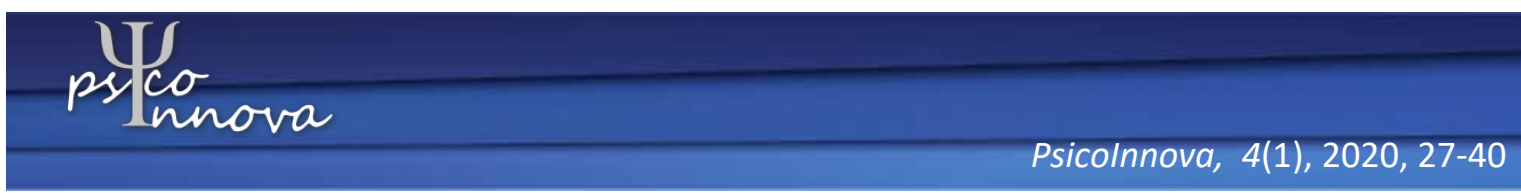




\section{Vivencias de Nuestra Comunidad Aprendiente.}

\subsection{Primera Vivencia: La Metamorfosis.}

Los cinco miembros de nuestra comunidad de aprendizaje hemos pasado por una serie de altibajos emocionales durante estos dos años y medio, tanto a nivel individual como a nivel grupal. Mientras cada integrante asimilaba la carga de lectura requerida para este doctorado, y la acomodaba con su diario vivir, a modo de una "travesía"; se iba gestando un proceso de metamorfosis o sea transformación individual y colectiva; y muchos de los párrafos leídos nos tocaban ciertas fibras y dejaban "huellas".

En los primeros cursos, cuando mirábamos el mural, de color azul y blanco, a la entrada del edificio del doctorado nos preguntábamos: - ¿iremos realmente a volar? (El mural dice: Acercaos al abismo, les dijo. Tenemos miedo, respondieron. Acercaos al abismo, les dijo. Se acercaron. Él les empujó... y salieron volando. - (Guillaume Apollinaire, citado por De Andrés y Andrés, 2011, p. 248).

La visión del doctorado es ser una alternativa para las personas que deben trabajar para lograr un sustento y no es un doctorado como en otras latitudes donde el aprendiente está dedicado tiempo completo, e incluso vive en el campus. Esto apelaba a nuestras necesidades, y si no hubiera sido de esta forma, muchos no nos hubiéramos matriculado. Durante la travesía, los cinco integrantes compartimos las carreras y congojas para pagar los aranceles, y estas preocupaciones, así como muchas otras, fueron derribando barreras de la intimidad personal y se fue creando más vínculo, más confianza. Un grupo conformado por cinco desconocidos y desconocidas quienes; primeramente, nos conocimos para autoorganizarnos y nos acoplamos. Esta interacción generó riqueza para poder ir entregando trabajos grupales, acompañarnos, compartir, lenguajear, promover reconfiguraciones semánticas, aprender y desaprender.

A nivel de prácticas académicas, este doctorado también es distinto. Es más, busca intencionadamente romper con estrategias educativas antiguas sin sentido y da paso a una experiencia nueva, cargada de pasión, emoción, y sentimiento. No hay clase explicativa del profesor tradicional sino una lectura intencionada de textos escogidos (Gutiérrez, 2006). El plan de estudios de este doctorado promueve un enfoque humanista, de desarrollo integral, sociocultural e interactivo entre los diferentes actores del proceso educativo. El programa doctoral se rige por tres principios: autoorganización, interdependencia y sostenibilidad y procura que los y las estudiantes "aprehendan" la realidad en su dimensión estética, ética y ecológica; que conozcan las características de la educación para el siglo XXI; que dominen los procesos y tratamientos de la mediación pedagógica; que estén al tanto de los nuevos paradigmas científicos; y que estén actualizados con las nuevas tecnologías de información (Gutiérrez y Prado, 2015).

\subsection{Segunda Vivencia: Sobre la Metodología Grupal.}

La metodología del doctorado se basa en crear comunidades de aprendizaje desde el primer curso, con la característica de que sean autoorganizadas y de que estén fuera del aula

\section{psco


de clases, de preferencia en lugares placenteros y variados. Esta situación fue también una experiencia nueva. Nadie nos tomó de la mano y nos explicó los pasos para autoorganizarse. En este doctorado no hay uniformidad, control externo, estabilidad y seguridad; sino, lo que promueve es la divergencia, la dinamicidad, la incertidumbre, la interacción, y la interrelación (Gutiérrez, 2006, p. 19). Nos dimos cuenta de que para no abdicar y renunciar era necesario "cambiar de mentalidad y de visión respecto a la autoridad, jerarquía, liderazgo, normas de convivencia, políticas y valores" (Gutiérrez y Prado, 2015). Es obvio que por más que la llama del fuego por lograr un doctorado nos anime, en la práctica y en el diario vivir, en cuanto a trabajos grupales, el grado de aporte de los miembros del grupo varía; así como el ritmo de lectura y comprensión; y el dominio y maestría de los temas. La ética de cada participante en este doctorado tiene un impacto importante en nuestra comunidad de aprendizaje.

La modalidad de entregar un ensayo grupal y uno individual durante la primera mitad del programa, nos obligó a conformar un grupo de trabajo para realizar las entregas grupales en tiempo y con la calidad esperada. La producción grupal no puede ser un conjunto de párrafos redactados por cada integrante, sino que debe ser un entretejido elaborado por todos y todas. Como en todo grupo de trabajo conformado por iguales, emergieron roles entre nosotros; curiosamente algunos se mantuvieron durante todos los cursos, otros se fueron rotando o cambiando de acuerdo con las circunstancias personales y a las del entorno. Para cada entrega, la comunidad Trascendencia se autoadaptaba y siempre logró su objetivo. Al conocer la Teoría de Santiago propuesta por Maturana y Varela (2006), e impregnarnos del concepto de "autopoiesis", comprobamos que el estar aprendiendo es signo de estar vivo y viceversa. Gutiérrez (citado por Díaz y Prado, 2015, p. 15) nos indican que "Educarse es impregnar de sentido la práctica de la vida cotidiana”. Díaz y Prado igualmente agregan que "necesitamos incorporar al proceso de vida lo que leemos, reflexionamos y compartimos" (2015, p. 9). Y es que también hemos entendido el mensaje del fundador cuando afirma que este es un doctorado del cual no se puede egresar: "ingresar para egresar es equivocarse de puerta" (Gutiérrez, 2006, p. 5). O sea, nuestro sentido de la vida es seguir aprendiendo.

\subsection{Tercera Vivencia: Configurando el Entramado en Convivencia.}

A nivel grupal, otra frase de Gutiérrez nos recuerda que "En la convivencia lo que interesa es el proceso de vivir" (citado por Díaz y Prado, 2015, p. 9). Podemos afirmar que dicho objetivo se cumplió en nuestra comunidad, ya que a todos y a todas nos "tocaron" las lecturas, las charlas, las películas y los talleres. Cada uno y cada una entró en mundos antes no conocidos, y quedó clarísima la diferencia entre la visión mecanicista y la nueva visión sistémica. Hemos pasado por un proceso de transformación, vemos la cotidianeidad de forma diferente y tenemos un mejor entendimiento del ser humano como especie.

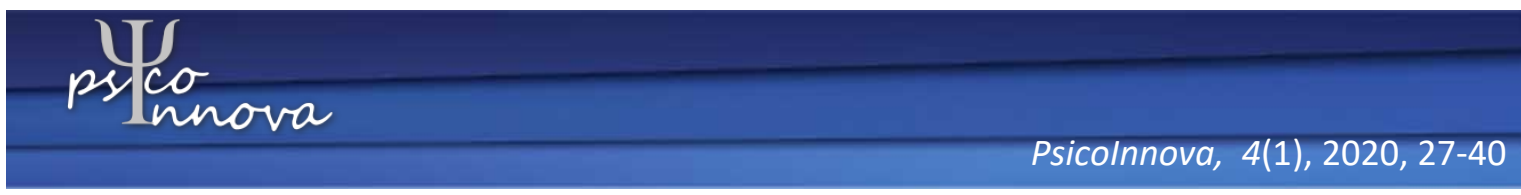


En nuestra comunidad de aprendizaje, cada integrante procuró leer y entender las lecturas asignadas en cada curso. Dentro de lo posible, todos y todas asistimos a las charlas que organiza la Universidad con distinguidos invitados e invitadas, asistimos y participamos a los talleres organizados por los y las tutoras, y además preparamos nuestros propios talleres. En los trabajos grupales que había que entregar en cada curso, los cinco miembros hicieron sus respectivos aportes, y el trabajo en grupo fue como el de los deportistas como maratonistas o ciclistas, donde entre todos y todas se van dando apoyo y nadie se queda atrás. La métrica no fue quién aportó más y quien aportó menos; sino que cada uno/a entregó lo mejor de sí. Sin querer, "la generación del conocimiento se reconfiguró sobre principios de colaboración, cooperación y solidaridad con el uno y la otra, [y] no de competencia y depredación" (Gutiérrez, 2006, p. 34). La vida no es constante, hay semanas con más trabajo en la oficina o en la escuela, hay problemas familiares, hay estrechez económica. Pero el doctorado fue creado para este tipo de personas, no para los que obtienen una beca por 5 años y se van tiempo completo a residir en un campus.

Fue precisamente esos mismos principios de colaboración, cooperación y solidaridad la que llevó a un integrante de nuestra comunidad de aprendizaje a dejar de avanzar con nuestro grupo. Pareciera que, en alguna parte del camino, los objetivos dejaron de ser compartidos, de converger los valores y aspiraciones de co-crear y convivir en el sueño de alcanzar la conclusión del programa doctoral. Pero más que ello, fue la sensación o vivencia de lo que indica Elizalde (2003), al mencionar el desafío de desarrollarnos (...)

(...) como seres éticos y asumir la responsabilidad por nuestro accionar en el mundo, y ser capaces de entender que nuestra calidad de vida alcanza su plenitud cuando trascendemos nuestra conciencia individual hacia una forma de conciencia capaz de sentir como propia, no sólo nuestra necesidad, sino además la de otro ser humano y de otra forma de vida (p.132).

En diversas ocasiones como comunidad, el caos e incertidumbre nos acompañó, desplazando el equilibrio dinámico; sin embargo, logramos reinventarnos y generar acciones en beneficio de todos y todas, sobretodo ante este integrante, con lo cual la situación cambió a pesar de los diversos esfuerzos por reencontrarnos con él también. Con mucha gratitud y humildad comprendimos que los caminos se separaban ahí.

Desde esta perspectiva, nuestra comunidad experimentó cómo al estar en permanente configuración del entramado en algunos momentos, puede darse la vivencia de que algún integrante deje de estar implicado con "la realidad" que se estaba entretejiendo, y casi que de forma "natural" deja de ser parte de la comunidad. Lo percibimos como si la misma autoorganización que se va gestando puede presentar este tipo de situaciones de exclusión de algún integrante.

\subsection{Cuarta Vivencia: Sobre el Núcleo Generador.}

Durante las distintas actividades doctorales, se planteaba un núcleo generador que nos movía desde nuestras personalidades, sentimientos, miradas, áreas profesionales,

\section{PSco


académicas y personales para entablar los encuentros, los lenguajeos, los ensayos, entre otras experiencias.

El proceso que sigue el núcleo generador nos remite a que una vez elaborado el mismo sigue su lanzamiento y estudio por medio de las tres lecturas: connotativa (subjetiva), denotativa (objetiva), y estructural (causas, razones, consecuencias). Gutiérrez (2010, p. 7) nos define la idiogenomatesis "como el aprendizaje concebido como un irse haciendo el ser humano por su propia iniciativa, como el propio generarse observando y leyendo los aconteceres de la capacidad de comprender y aprender". Su relevancia subyace en cuanto a que ésta, junto con el núcleo generador son el camino a la expresión creadora. Ya que el significado no se encuentra en el objeto de estudio, sino que depende de sus significantes, o sea de las miradas del observador y sus interpretaciones.

Nuestro conocimiento del universo se deriva de este acto de participación en el que estamos implicados nosotros y nosotras, nuestros sentidos, los instrumentos que utilizamos en los experimentos, la manera en que nos comunicamos y las vías que escogemos para descubrir la naturaleza. Así pues, este conocimiento es al mismo tiempo subjetivo y objetivo. Para que surgiera el conocimiento, que dejamos plasmado en cada uno de los talleres, blogs, sociodramas, ensayos y otros como una experiencia creadora, lo que necesitábamos era un juego libre entre las y los miembros de la comunidad, de modo que la mente no se viera sometida en ningún momento de manera rígida a un conjunto limitado de presupuestos.

Ciertamente, las emergencias y aprendizajes significativos los construimos en conjunto. Sólo cuando la inteligencia opera de manera libre y creativa puede la mente abandonar las estructuras de categoría rígidas y por tanto comprometerse en la formación de órdenes nuevos. Por ejemplo, cuando desarrollamos en comunidad un taller autoorganizado bajo el eje generador del poder, nos reunimos múltiples veces con el propósito de recabar en comunidad nuestras diferentes miradas sobre el concepto de poder. Aunque al principio el concepto fue planteado como un constructo social basado en el control, generalmente por las clases dominantes, luego, después de varias horas de diálogo, transformamos el concepto de poder como aquel que cada persona posee y que puede ejercer desde su interior, para ejercer un impacto positivo en el exterior. Este tipo de poder fue plasmado durante el taller bajo las premisas de amor al prójimo, convivir sanamente todos y todas, y meditación para reconectarnos con nuestro ser interior. 


\subsection{Quinta Vivencia: Sobre los Círculos de Cultura y Diálogo.}

Cuando entramos a este programa doctoral veníamos en busca de una base epistémica crítica, con la intención transformadora de llevar la emancipación a todas nuestras esferas y realidades humanas. Como dice el proverbio chino: dadle a una persona y comerá por un día, enséñale a pescar y comerá por toda su vida. Este tipo de pedagogía crítica que estamos abordando, se contrapone al adoctrinamiento mecanicista y determinista de nuestro sistema educativo actual. A su vez, tiene como principio transformar el mundo y emancipar el ser humano, el cual debe también transformarse. Freire nos decía sabiamente "todo acto educativo, es un acto político". Creemos que no se equivocó, dado que al llevar este doctorado hacemos política; aunque esta vez, una política crítica, problematizadora y tomando en cuenta las éticas que han sido degradadas por nuestra sociedad globalizada.

Los círculos de cultura nos hicieron crecer como comunidad en sus tres fases, la primera nadie lo conoce todo, la segunda, todos y todas contribuimos a multiplicidad de saberes, y la tercera, estos saberes en conjunto lograron provocar emergencias problematizadoras, que nos llevaban a un núcleo generador del cual, sobrevienen diferentes perturbaciones. Estas perturbaciones nos tomaban de la mano a través de largas horas de diálogo del que emergen nuestras propuestas restaurativas en nuestro entorno real como seres humanos. La humildad y la confianza nos permiten en este sentido la configuración de una postura amorosa, desde la cual el diálogo se hace humanizador, vinculante, potencializador de lo humano en sus dimensiones de racionalidad, apertura, y libertad. Aprendimos de esta manera, que el diálogo es un encuentro amoroso donde el ser humano, junto con otros y otras, transforma el mundo y humanizando, se humaniza a sí mismo.

Durante los diversos espacios de diálogo en comunidad aprendiente que tuvimos en este doctorado, tomó significado para todos y todas, la conocida frase de Freire: "nadie libera a nadie, nadie se libera solo. Los humanos nos liberamos en comunión". Este compartir en comunión, nos mostró la importancia de sacar los conocimientos y saberes de las cuatro paredes de la educación formal y llevarlo a espacios más libres como nuestros encuentros con familiares, amigas y amigos. Ciertamente, la emancipación humana, sólo es posible como forma de vida, como una manera de habitar el mundo. Lo ético y lo estético en la emancipación es un hacerse responsable con la propia existencia y la de nuestro entorno.

\subsection{Sexta Vivencia: Sobre las Valoraciones.}

Dado que una de las formas de valoración que sugiere el Dr. Gutiérrez, es que el o la aprendiente narre, escriba o cuente lo que aprendió, la metodología de evaluación ha consistido en lo siguiente: redactar un ensayo individual; redactar un ensayo grupal; redactar y publicar un blog; redactar un texto paralelo; realizar un taller entre las y los miembros de la comunidad; asistir y participar en los talleres que organizan los y las tutoras, y asistir a las charlas y conversatorios que organiza la universidad. Cada actividad es evaluada por el o la tutora y en el caso de los talleres por el equipo de tutores asignado a cada generación.

\section{psto


Respecto al sentido de la evaluación menciona Gutiérrez (2006, p.83):

en primer lugar se debe tener presente la importancia del contenido de cada curso que le permitirán al aprendiente encontrar la razones y los porqués, los fundamentos, principios y valores del paradigma actual deben ser reemplazados por los fundamentos, principios y valores del paradigma emergente

Y sigue: "El segundo elemento fundamental del proceso de evaluación es la comprobación de la presencia de la chifladura en la lectura. No se trata de comentar a los autores de los cursos, sino de verlos desde la intencionalidad propia” (Gutiérrez, 2006, pág. 83)

Las comunidades de aprendizaje son escenarios "ideales" para reconfigurar, cogestar y co-crear las realidades situadas de cada contexto, en donde las vivencias y aprendizajes de vida de cada persona son relevantes. Esta modalidad de evaluación puede facilitar que cada integrante realice sus propias reflexiones a nivel individual y paralelamente con su comunidad, de forma que en interacción permanente consigo mismo y con los demás; pueda ir resignificando y construyendo el tejido social del cual se es parte activa.

Es probable que esta forma de ser realimentados o bien valuados permitió que fuéramos descifrando un lenguaje propio como comunidad. Los diálogos y lenguajeos culminaron en producciones escritas como una memoria colectiva de cómo en comunidad nos tornamos más sensibles y conscientes ante el tejido social y las diversas miradas complejas en las cuales nos visualizamos como seres implicados.

\subsection{Sétima Vivencia: Sobre el Texto Paralelo.}

Podemos partir de la premisa de que la realidad tiene una estructura con varios niveles de observación relevantes. Cada nivel de observación induce una subrealidad con sus propias leyes, una subrealidad con su propia subconstitución. La estructura de la realidad está compuesta entonces de realidades que se contienen unas a las otras como las famosas muñecas rusas. El texto paralelo, en este sentido, es como un instrumento orientador para elaborar teoría y accionar en la práctica, permite reflexionar para la construcción del conocimiento. El texto paralelo integra la teoría y la práctica hacia un acto crítico, realista y racional.

El texto paralelo, no debe verse únicamente como una actividad manual, creemos que es mucho más que eso, es el interlocutor presente en todo momento que acepta el interrogatorio, la narración, el cuestionamiento, la búsqueda de la información y un sin fin de técnicas pedagógicas que conducen al descanso y a la relajación, desarrollando procesos mentales que van de la mano al cerebro, de lo concreto a lo abstracto, de la práctica a la teoría y viceversa (Gutierrez, 2010, pág. 10). 
En el trabajo en comunidad, el texto paralelo sin duda alguna nos permitió ser partícipes de la realidad social, cultural y política, dado que el conocimiento lo adquirimos de la lectura del contexto de distintos autores y autoras y lo relacionamos con nuestra práctica y quehacer diario. Nuestra comunidad tuvo la oportunidad de crear varios textos paralelos en forma conjunta, uno de ellos fue sobre el tema de las características educativas en el siglo XXI. Para este texto paralelo realizamos la lectura de algunos autores y autoras relevantes en los temas de biopedagogía y complejidad y diseñamos como producto visual una revista. Esta revista logró compilar varios artículos escritos por los miembros de la comunidad bajo la luz de los textos de "El Campo Akásico" y "Las Siete Leyes del Caos" y el resultado fue una serie de emergencias colectivas que sin duda alguna ayudaron a que la comunidad madurara y se fortaleciera en la discusión de las características educativas que queremos para nuestros hijos e hijas y las futuras generaciones.

\subsection{Octava Vivencia: Sobre el Uso de las Tecnologías.}

La sociedad humana se desarrolla y despliega, interactuando, relacionándose y accionando interconexiones metodológicas que requieren el uso de pensamiento, conocimiento, lenguaje y simbología. Para nuestra comunidad ha sido un recurso el uso de las tecnologías en el colaborar y compartir de los diferentes intereses, tareas y objetivos que perseguimos conjuntamente, accediendo a que la información proporcionada sea diseñada dentro de un ambiente de interacción social en el cual todos y todas somos actores que nos sobreponemos al ambiente físico, facilitando la comunicación entre las personas que habitan en diferentes espacios geográficos y que aportan a su vez diversidad cultural de los y las participantes que integran múltiples herramientas tecnológicas en la transferencia de conocimiento, a una velocidad que nuestros antepasados nunca antes soñaron, todo esto bajo la perspectiva del trabajo colaborativo entre las y los miembros de la comunidad que basan el uso de las diferentes tecnologías en problemas, resolución de casos y círculos de aprendizaje, potenciando sus ideas y conocimientos (Cabero, 2006)

Estos instrumentos tecnológicos pretendemos hacerlos un recurso de apoyo ya que nos permitieron diseñar textos paralelos, talleres, blogs y un sin fin de ideas y pensamientos que invita el asumir y cumplir con los compromisos grupales. Así mismo acortaron distancias sin importar lo lejos que se encuentren los unos y las otras, siempre se está en contacto, realimentando, construyendo, colaborando y fluyendo como una sola célula. Más sin embargo, el desarrollo de las competencias del cooperar y compartir con los amigos, amigas y colegas en reuniones presenciales, departiendo alrededor y calor de una mesa, una comida o un café es algo precioso, es un tiempo y espacio que no tiene precio y que la tecnología no puede sustituir aún, ya que es lo que emana de los diferentes sistemas de seres vivos.

El pensar desde una realidad que enlaza el pensamiento de los y las aprendientes en su manera específica y particular de entender el mundo, su historia y visión, proporciona diversas corrientes de pensamiento y conocimiento compartido, que permiten a sus protagonistas en forma epistémica liderar la sensibilización entre sus participantes de la comunidad aprendiente, mediante la toma de decisiones, selección de prioridades 
compartidas y estrategias de logro en la construcción colectiva del conocimiento social y de presencia cognitiva, que se da por medio del pensamiento y hacer comunitario. No sólo como comunidad aprendiente, sino generacional de doctorandos e interdisciplinaria de la universidad, en alguna medida nuestra comunidad de aprendizaje supo hacer un buen uso de los sistemas virtuales ya que aportan accesibilidad tecnológica para colaborar culturalmente en la diversidad de las vivencias comunales.

Estas constituyen la clave fundamental en el flujo de información que desarrolla destrezas comunicativas, informativas y de procesamiento e intercambio con otros y otras como por ejemplo la oportunidad de compartir con invitados internacionales como el doctor Maldonado. Sus charlas fueron compartidas en la plataforma virtual universitaria para aquellos y aquellas que no les fue posible asistir. Así mismo, los miembros de la comunidad tuvimos la colaboración y participación en proyectos y talleres intercomunitarios, debates en línea en dónde; por ejemplo, nos enriquecemos con los puntos visionarios de la doctora Dennise Najmanovich. Este recurso tecnológico ha permitido a nuestras comunidades gozar de una gran flexibilidad, reduciendo la brecha de la separación temporal y/o espacial de sus integrantes como lo es el caso de los compañeros y compañeras provenientes de zonas fuera del Gran Área Metropolitana, Colombia, Panamá, Nicaragua, o Qatar.

\subsection{Novena Vivencia: Importancia del Debate sobre un Eje Generador.}

Al comienzo del doctorado, la mayoría de las y los integrantes de nuestra comunidad no conocíamos los tipos de inteligencia artificial (IA) como medios de información y mediación pedagógica sea esta teórica o práctica. Se conocía el uso de las tecnologías, pero no cómo la inteligencia artificial ayuda a los miembros de grupo a experimentar vivencias de aprendizaje, construcción de conocimiento y debate con otras personas ajenas a la comunidad. La inteligencia artificial ha sido un tema de debate entre la ciudadanía en general pero principalmente entre docentes. Y nuestra comunidad de aprendizaje no fue la excepción. Los motivos son variados, por ejemplo, compartimos sentires en cuanto a la posible sustitución de la inteligencia artificial por la mano de obra humana. Unos pensamos que sí va a suceder y otros compañeros que sólo va funcionar como un colaborador más para los y las profesionales en diferentes campos como la medicina, la industria vehicular, educación, entre otros.

Por otro lado, todos llegamos a un acuerdo en cuanto al beneficio de algunos pocos para la elaboración de la inteligencia artificial. Los grandes capitalistas se están aprovechando de que cada día se emplea más este tipo de inteligencia para hacer más "fácil o rápida" algunas tareas que solíamos realizar con mayor tiempo. Claro está, que más inteligencia artificial implica la destrucción del ambiente de una forma apresurada, inconsciente y devastadora para la flora y fauna. Sin embargo, esto parece no importarle a nadie.

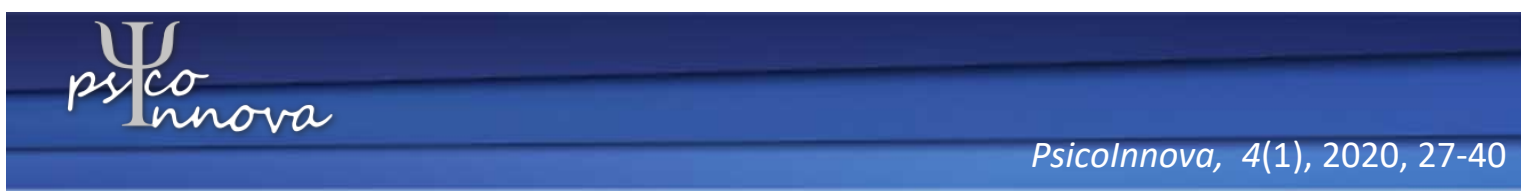


La inteligencia artificial en nuestra comunidad de aprendizaje no ha sido utilizada al $100 \%$ ya que aún se está trabajando para mejorar la autoorganización de las máquinas, el entrenamiento máquina a máquina (coaching), la estética (forma humana, aunque no necesaria), y sentimientos. La inteligencia artificial es un sistema complejo y por qué no caótico para desafiar a la humanidad en cuanto al uso del razonamiento, construcción de aprendizaje y sentido crítico.

Gracias a esta vivencia, nuestra comunidad fue capaz de dialogar sobre muchos temas que hemos estudiado en este doctorado y compararlo con las ventajas y desventajas que poseen las inteligencias artificiales. Si en una comunidad no existen desacuerdos, discusiones sanas y puntos de vista distintos, el lenguajeo no tendría sentido. El respeto y la empatía se llevaron a cabo con éxito en esta comunidad aprendiente. Tal vez no aplicamos la inteligencia artificial en nuestra comunidad como prioridad, pero si somos conscientes que fue, en nuestro caso, una herramienta muy valiosa para generar diálogo, conocimiento, incertidumbre y perturbaciones iónicas.

\section{Conclusiones}

Finalmente, después del camino que vivimos y compartimos como comunidad de aprendizaje durante el doctorado universitario en Educación de la Universidad La Salle de Costa Rica, intentamos brindar algunas conclusiones que permitan dejar al descubierto algunas de las bondades y alcances de las comunidades de aprendizaje, las cuales nos apasionan y con las cuales esperamos también desarrollar este mismo sentimiento en las futuras generaciones.

\section{¿Por qué comunidades de aprendizaje?}

Consideramos que desde las comunidades de aprendizaje se pueden co-crear formas alternativas de autoorganización en donde el diálogo incluyente se convierte en una forma valiosa de mediar y lograr entramados comunitarios en los cuales se resignifiquen prioridades y necesidades al aprender, sin perder la reciprocidad, la solidaridad, la posibilidad de apropiación de este proceso con identidad cultural.

Podría resultar paradójico nombrar a las comunidades de aprendizaje como metodología y/o técnica alternativa para construir y generar soluciones culturalmente sensibles a las necesidades que se presentan al aprender o al conocer, pero realmente si lo analizamos bien y sin querer caer en reduccionismos o recetas de enseñanza, las comunidades de aprendizaje mediadas con el fin de desarrollar trabajo solidario, humano, en beneficio de los y las integrantes de la comunidad, es una oportunidad, una alternativa para hacer camino hacia una educación reinventada, implicada en el tejido social que hoy parece ser un sueño; esa utopía que pretende gestar nuevos escenarios sociales y decolonizar la forma en que se aprende.

\section{¿Cuál es la importancia de construir espacios biocráticos como comunidades aprendientes en diferentes contextos?}

Las comunidades de aprendizaje deberían tener como uno de sus objetivos el ofrecer a la persona potencializarse como ser humano integrador, responsable con su entorno,

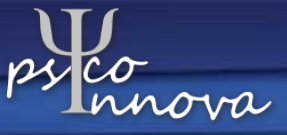


dinamizador de transformaciones, problematizador de experiencias de aprendizaje, crítico y en sintonía con el universo. Todas estas son habilidades para vivir que nos han sido arrebatadas a la luz de la globalización y la democracia donde nos atan como sujetos de sociedad donde la voz y el voto ha sido reservado, algunas veces comprado y pagado para algunas élites relegando la otredad en la exclusión. Los y las docentes tenemos un gran reto, el de actuar como agentes dinamizadores y promotores de acción social descolarizando espacios y saberes que han sido arrebatados a los pueblos -- desde ahí se construye la biocracia.

De esta manera, las comunidades de aprendizaje poseen potencial para disminuir la brecha entre las dicotomías vertical y horizontal, ricos y pobres, blancos y negros, hombres y mujeres, y nos dan núcleos generadores integradores de problemáticas colectivas para resolver en comunión. Bajo la práctica de nuevas formas de convivencia, nos damos cuenta de que el conocimiento y el saber ya no son monopolizados por pequeños grupos elitistas, escolarizantes, y doctrinantes llamados, escuelas, colegios y/o universidades; sino más bien, que los saberes planetarios fueron originarios de nuestros antepasados indígenas quienes crecían y vivían en entramados sociales de biocracia, a lo cual se debería retornar.

En sí, este trabajo de investigación constituye en toda su base una propuesta en busca del bien común por medio del tejido de la comunidad de aprendizaje, las cuales no deben de quedarse en los centros educativos, sino que deben de derrumbar muros y empoderar a otros grupos sociales. Esperamos que, al comienzo de esta nueva Era, la Era del Gran Giro, que quebranta paradigmas de educación tradicionalista, abra sus puertas a la distribución y construcción equitativa de saberes donde los pueblos sean integrados a trabajar en comunidades aprendientes y las ontologías coloquiales se pongan a dialogar en biocracia con antiguos saberes cientificistas.

\section{¿Cómo podrían resonar y sinergizar las universidades en la transformación y en la decolonización del saber?}

Hemos escuchado en diversas ocasiones que no se puede dar lo que no se ha recibido. En este sentido experimentar ser comunidad de aprendizaje, la identidad y la pertenencia que desde cada una de ellas se puede desarrollar, así como la práctica del diálogo comprometido, de ser solidario, de interactuar respetuosamente, logrando hacer "realidad" la capacidad de autoorganizarse en beneficio del grupo y no de mi interés o de pocos, desde las universidades, puede permitir desarrollar convicción en esta manera de releer las diversas miradas y opciones de solución ante las causas comunes que se define interactuar.

Por lo que, poder transmitir estas experiencias, visualizarlas y socializarlas desde la universidad, la cual cuenta con la plataforma para ello, favorece la posibilidad de darle un lugar al multiculturalismo, a la diversidad, que nos invita a abrir los ojos, la mente, y el corazón a la diversidad de opciones y alternativas que se pueden co-crear y cogestar como unidad, evitando caer en dualidades u opciones únicas, al buscar resolver diferentes necesidades presentes en los procesos de educación. Poder divulgar toda esta experiencia es una forma de aportar al conocimiento que parte de las comunidades de aprendizaje.

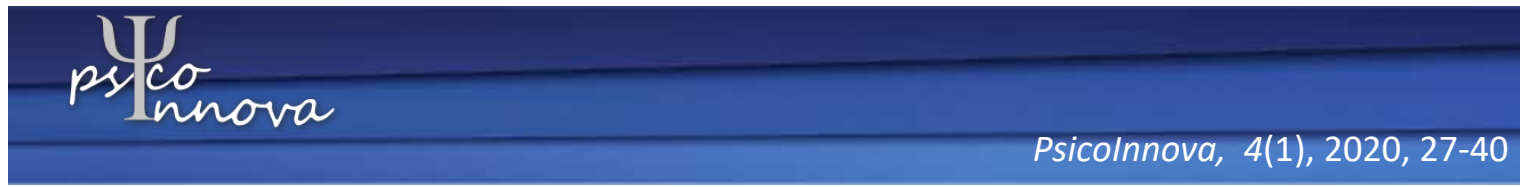


Los escritos académicos son necesarios para compartir las vivencias y el conocimiento que se van experimentando y no convertirse en fábricas de publicaciones que solo siguen las normas de publicación en nombre de la evidencia científica. Esto es una de las tantas formas de compartir lo aprendido y lo que se va construyendo, lo que se siente y se piensa sobre prácticas sociales y educativas que desean transformar y aportar a las miradas alternativas a las ya instituidas. Definitivamente consideramos que ello se convierte en una forma de decolonizar el aprendizaje, sus fundamentos y razón de ser.

Las universidades, desde las vivencias de comunidad de aprendizaje, pueden procurar que al co-razonar las reflexiones que se gestan, permitan reinterpretar y rearticular necesidades e inquietudes sociales y educativas que no hemos logrado "superar" dentro del sistema educativo. Cada día, de alguna manera, está permitiendo y hasta contribuyendo con que la brecha de la exclusión social sea más grande, ya que el ser humano desconoce su responsabilidad y su capacidad para sentipensar y dejar emerger el "poder de", así como apropiarse de sus necesidades para alzar la voz y proponer acciones que permitan avanzar en la resolución de estas condiciones.

\section{información?}

¿Cuál es el reto de las comunidades de aprendizaje y las nuevas tecnologías de la

Partimos y creemos firmemente que la tecnología no podrá sustituir el aprendizaje en el ser humano, más sí podría ser un complemento a los contactos que experimentamos presencialmente. La tecnología no sustituye al ser humano, pero puede aportar al aprendizaje, máxime en los contextos universitarios, sin querer excluir los otros escenarios en los cuales puede ser un recurso de apoyo, un medio, más no convertirse en un fin en sí mismo. Pareciera que uno de los grandes desafíos que nos presenta este siglo XXI es cómo, sin perder el valor de la interacción, de la comunalidad, cara a cara, del diálogo, sin atropellar las prácticas sociales y educativas efectivas, podemos establecer condiciones para que la tecnología esté al servicio del ser humano. Precisamente este desafío puede ser tema y necesidad que se puede repensar, reflexionar en comunidades de aprendizaje universitarias, de forma que nos acerque cada vez más a la posibilidad de pensar multidimensionalmente.

\section{aprendizaje?}

¿Hacia dónde nos dirigimos con la inteligencia artificial en los entornos de

Reconocemos como seres humanos la necesidad de estar actualizados y ser conscientes interactuantes con los sistemas complejos que surjan. Dejar de lado los sistemas inteligentes, entre ellos, la inteligencia artificial, sería un acto despreocupado por parte de los y las miembros de las comunidades. En este sentido, no debemos resistirnos al cambio, sino adaptarnos para conseguir cambios significativos en el aprendizaje. Sin embargo, no ignoramos los riesgos que representan en cuanto las grandes transnacionales bombardean y presionan para que nos sumerjamos en el mundo tecnológico que nos vende la globalización. Un ejemplo de la grave amenaza que visualizamos y vivimos actualmente es el robo de nuestros datos biométricos por algunas empresas inescrupulosas que desean tener el control por sobre el bien común.

\section{PSco




\section{Referencias}

Cabero, J. (2006). Comunidades Virtuales para el Aprendizaje. Su Utilización en la Enseñanza. Edutec.

Castillo, R. y Ramírez, P. (2016). Semilleros de Investigación y Comunidades Aprendientes: Una experiencia para Cosechar Oportunidades. Red Pensar, 5(1), 109-112. https://ojs.redpensar.ulasalle.ac.cr/index.php/redpensar/article/view/102

Coll, C. (2001). Simposium Internacional sobre las Comunidades de Aprendizaje. Barcelona: Fórum Universal de las Culturas Barcelona-2004. [Documento No Publicado]. http://www.tafor.net/psicoaula/campus/master/master/experto1/unidad16/images/ca. pdf

De Andrés, V., y Andrés, F. (2011). Confianza total para vivir mejor. (1 ${ }^{\text {a }}$ Ed). Buenos Aires: Planeta

Elizalde, A. (2003). Desarrollo humano y ética para sustentabilidad. Chile: Universidad Bolivariana

Gutiérrez, F. (2010). Las Nuevas Ciencias de la Vida. Polis, 9(5), 223-233. https://scielo.conicyt.cl/pdf/polis/v9n25/art12.pdf

Gutiérrez, F. (2006). Doctorado de la tercera cultura. En busca del sentido. San José: Nuestra Tierra.

Gutiérrez, F., y Prado, C. (2015). Ecopedagogía y Ciudadanía Planetaria. México: De La Salle ediciones.

Gutiérrez, F. y Prieto, D. (2002). La Mediación Pedagógica. Madrid: Xátiva.

Harari, Y. (2018). Sapiens. De Animales a Dioses: Una breve historia de la humanidad. México: Penguin Random House Grupo Editorial.

Maturana, H. y Varela, F. (2006). De Máquinas y Seres Vivos: Autopoiesis: La Organización de lo Vivo. España: Editorial Universitaria Lumen 